\title{
Aproximación al estudio de la visibilidad de la prensa local y regional en Internet: el análisis de las metatags
}

\author{
Àngels Jiménez \\ Eulàlia Fuentes \\ Alfons González \\ Área de Documentación. Facultad de Ciencias de la Comunicación. \\ Universidad Autónoma de Barcelona (España)
}

\subsection{Resumen}

Se analiza el uso y el contenido de las metaetiquetas de una muestra representativa de diarios locales y regionales españoles. Se dedica una atención especial a las metaetiquetas 'description' y 'keywords'.

Palabras clave: Metaetiquetas. Prensa digital.

\subsection{Abstract}

The use and the content of the metatags of a representative sample of local and regional Spanish newspapers is analyzed. A special attention is dedicated to the metatags 'description' and 'keywords'.

Keywords: Metatags. Digital press.

\section{Introducción}

Desde 1995, año en el que aparecen las primeras versiones digitales de diarios locales y regionales en Internet, con el catalán Avui a la cabeza, su nivel de presencia ha experimentado un crecimiento constante hasta superar ya el centenar de títulos, como refleja la figura 1 .

La prensa local y regional constituye el mejor ejemplo del denominado periodismo de proximidad. El área geográfica en la que desarrolla su actividad informativa y en la que mayoritariamente se distribuye y, en algunos casos, la lengua utilizada permiten caracterizar a este tipo de prensa impresa y distinguirla de la de alcance nacional. Son rasgos que cabe interpretar como inherentes a la esencia de este tipo de prensa y que suelen traducirse cuantitativamente en niveles tanto 


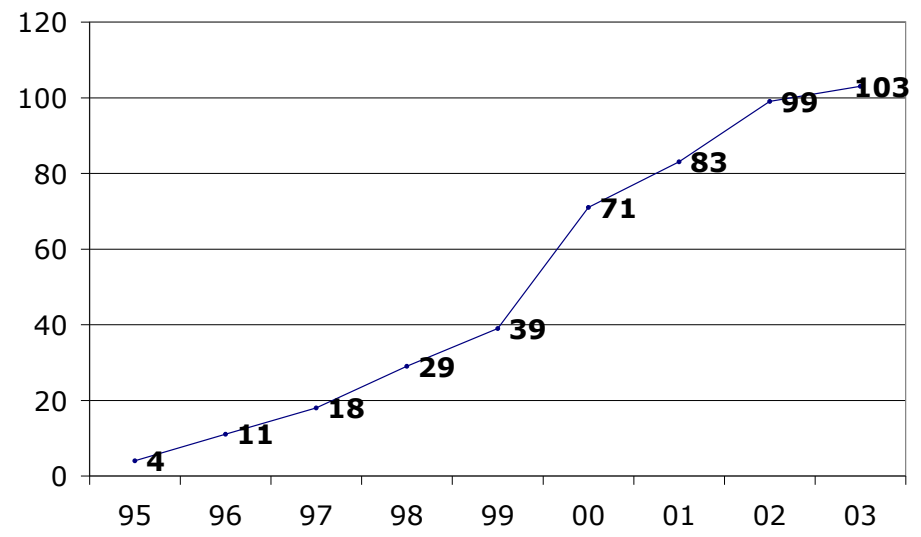

Figura 1. Evolución del número de versiones digitales de la prensa española local y regional [Fuente: elaboración propia]

de tirada como de difusión inferiores a los diarios de alcance nacional. Si bien es cierto que estos rasgos son propios del medio impreso, en teoría las ediciones digitales de la prensa local y regional no deberían estar influidas necesariamente por las inercias de la prensa impresa. El hecho de distribuirse en un entorno digital diluye parte de las limitaciones que ponen freno a su difusión en la Red.

La incorporación de los medios de comunicación en Internet, en conjunción con las propiedades de la información digital, ha propiciado la extensión de una serie de rasgos que constituyen un perfil nuevo para la prensa diaria como son la actualización constante de los contenidos, la distribución masiva de información sin limitaciones espaciales ni temporales, o la personalización. Junto a estas características existen otras en las que de forma directa o indirecta intervienen los procesos documentales para enriquecer la dimensión informativa y comunicativa de los medios: el acceso a información retrospectiva mediante la consulta de hemerotecas digitales, fototecas o archivos audiovisuales, y la consulta de bibliotecas de referencia virtuales constituidas por dossieres temáticos, directorios especializados o colecciones de datos estadísticos son algunos ejemplos de los nuevos servicios de información que los medios digitales proporcionan a sus lectores y al resto de usuarios de la Red.

Este nuevo perfil no es privativo de las cabeceras de renombre nacional o internacional. Si podemos hablar de la constitución de portales informativos al referirnos a las sedes web de medios como Le Monde, Washington Post o El Mundo, podríamos hacer lo mismo al referirnos a portales de información local en relación con diarios de ámbito más reducido. En ciertos casos estos rotativos se han convertido en portavoces de una realidad cultural o lingüística 
que difícilmente podía gozar de un canal más amplio y efectivo de difusión, y no sólo a través de la información puntual sobre el día a día de su entorno, sino mediante la elaboración de productos y servicios específicos - guías turísticas, dossieres temáticos, diccionarios biográficos, directorios de empresas, etc. - que trascienden la mera función informativa diaria. En esta nueva situación el perfil de la audiencia de estos medios no tiene porqué ser idéntica a la de sus ediciones impresas. En un contexto global como el de Internet el número de lectores de un diario no depende ya, entre otros, de factores como la tirada y la distribución geográfica de cada edición, sino de la calidad de sus productos y servicios informativos y del aprovechamiento de las ventajas tecnológicas que Internet y la información digital proporcionan. Es en este contexto en el que debemos hablar de la visibilidad de los medios de comunicación en la Red.

\section{Objetivo}

El Área de Documentación de la Universidad Autónoma de Barcelona está llevando a cabo una investigación encaminada a analizar la visibilidad de la prensa local y regional española en Internet. Para ello se ha previsto el estudio de una serie de parámetros para conocer el nivel de visibilidad de las sede web de los medios digitales. Los parámetros son cinco: volumen de información suministrada, difusión, diversificación y calidad de los productos y servicios informativos, luminosidad, y nivel de autodescripción. En este trabajo se ofrecen los resultados correspondientes al último criterio. Así pues, el propósito de este artículo es mostrar los resultados obtenidos en el análisis de la autodescripción efectuada por la prensa digital de ámbito local y regional.

\section{Objeto de estudio y metodología}

Del centenar de diarios de alcance local y regional en Internet se ha escogido una muestra representativa. El criterio aplicado ha sido seleccionar los diarios cuyas versiones electrónicas sean controladas por la Oficina de Justificación de la Difusión (OJD), ya que los datos que ofrece esta entidad constituyen otro de los parámetros a analizar en una fase posterior de este estudio. De este modo el universo de análisis se ha centrado en las sedes web de 23 diarios. El análisis del nivel de autodescripción de las sedes web se ha llevado a cabo a partir de la revisión del contenido de las etiquetas META o metatags.

La simplicidad del lenguaje HTML ha contribuido al enorme crecimiento de Internet, y aunque la función básica de este código sea la de describir la apariencia formal de las páginas en la Red, dispone también de una sintaxis propia para asignar información sobre los documentos web. Se trata de las metatags. Éstas se ubican en la cabecera del documento (HEAD), son invisibles a los navegadores y se utilizan para que las páginas de una sede web sean indizadas por los buscado-

Scire. $10: 2$ (jul.-dic. 2004) 99-114. 
res. Las metatags identifican propiedades del documento y asignan valores a esas propiedades, pero no existe un estándar que determine un conjunto de propiedades. La simplicidad del HTML para la publicación de documentos web explica que los autores no sigan un modelo normalizado de descripción que no vaya más allá de un conjunto de términos en lenguaje natural y en su propia lengua completando las etiquetas 'keyword' y 'description', que son las que mayoritariamente han tenido en cuenta los motores de búsqueda generales para indizar las páginas web y para calcular su relevancia.

En la mayoría de los casos el uso de metainformación ha tenido como objetivo aumentar la visibilidad en los motores de búsqueda. Pero el abuso que se ha

\begin{tabular}{|l|c|c|}
\hline \multicolumn{1}{c}{ Metatag } & $\mathbf{2 0 0 2}$ & $\mathbf{2 0 0 3}$ \\
\hline Audience & 1 & - \\
\hline Author & 3 & 1 \\
\hline Basis & 1 & - \\
\hline Cache-control & 1 & 1 \\
\hline Classification & 2 & 1 \\
\hline Copyright & 2 & 2 \\
\hline Description & 15 & 15 \\
\hline Distribution & 4 & 2 \\
\hline Document-rating & 1 & 1 \\
\hline Document-state & 2 & 1 \\
\hline Document-type & 1 & 1 \\
\hline Geo.placename & 1 & - \\
\hline Geo.region & 1 & - \\
\hline Keywords & 15 & 15 \\
\hline Language & 4 & 3 \\
\hline Locality & 1 & 1 \\
\hline Note & 1 & - \\
\hline Organization & 1 & 1 \\
\hline Origen & 1 & 1 \\
\hline Page Topic & 1 & - \\
\hline Rating & 1 & - \\
\hline Revisit & 1 & 1 \\
\hline Robots & 1 & - \\
\hline Seccion & 1 & \\
\hline Subjects & 1 & 1 \\
\hline & 1 & 1 \\
\hline & 1 & 1 \\
\hline & 1 & 1 \\
\hline & 1 & 1 \\
\hline
\end{tabular}

Tabla I. Tipo de metatags presentes en el elemento HEAD de las sedes web de los diarios locales durante los años 2002 y 2003 [Fuente: elaboración propia].

Scire. $10: 2$ (jul.-dic. 2004) 99-114. 
llegado a hacer de estas etiquetas (spamming) ha conducido a que cada vez sean más los buscadores que las hayan ignorado. A pesar de esta tendencia un buen número de diarios las ha actualizado en diversas ocasiones a lo largo del período 2001-2003. En este análisis no se pretende valorar el uso de las metatag para mejorar el posicionamiento de los medios en la Red, sino estudiarlas como una herramienta aplicada por los propios medios para su autodescripción.

\section{Medios con metaetiquetas}

El primer paso ha consistido en conocer cuántos de los medios seleccionados han incluido en su cabecera algún tipo de metatag. De los 23 diarios, 7 (Levante, Correo gallego, Diario de Cádiz, Diario de Burgos, Diario de Navarra, Diario de Noticias y el Heraldo de Aragón) no cuentan con ninguna. En sus cabeceras sólo han definido el elemento 'title'. Los 16 diarios restantes, que constituyen el objeto de análisis de este estudio, a pesar de haber incluido metatags en su cabecera presentan notables diferencias. Los datos sobre el número y el tipo de metatags utilizadas en el bienio 2002-2003 por los diarios locales estudiados se presenta de forma sintética en las tablas 1 y 2.

A partir de los datos observados conviene señalar algunos detalles significativos. En primer lugar, el número de metatags distintas utilizadas ha oscilado de las 27 en 2002 a las 19 en 2003. A pesar de la sensible reducción - consecuencia de los cambios en la composición del elemento HEAD de los diarios Comercio

\begin{tabular}{|l|c|c|}
\hline \multicolumn{1}{|c|}{ Diario } & $\mathbf{2 0 0 2}$ & $\mathbf{2 0 0 3}$ \\
\hline Avui & 1 & 1 \\
\hline Canarias 7 & 5 & 5 \\
\hline El Correo digital & 2 & 2 \\
\hline DiarioVasco.com & 2 & 2 \\
\hline Diario Sur digital & 2 & 2 \\
\hline El Norte de Castilla & 2 & 2 \\
\hline La Rioja.com & 2 & 2 \\
\hline El Comercio digital & 5 & 2 \\
\hline Faro de Vigo & 5 & 4 \\
\hline Ideal digital & 2 & 2 \\
\hline El Diario Montañés.es & 2 & 2 \\
\hline La Nueva España & 19 & 2 \\
\hline La Voz de Galicia & 7 & 7 \\
\hline Las Provincias digital & 2 & 2 \\
\hline
\end{tabular}

Tabla II. Distribución del número de metatags por diario durante los años 2002 y 2003 [Fuente: elaboración propia].

Scire. 10 : 2 (jul.-dic. 2004) 99-114. 
digital, Faro de Vigo y La Nueva España-, ambas cifras ponen de manifiesto la ausencia de normalización del código HTML a la hora de seleccionar el tipo de metatags. Este dato se corrobora también si se tiene en cuenta la distribución de etiquetas por diario: sólo la mitad de los estudiados coinciden en el número y tipo de metatags seleccionadas. Por último, cabe indicar que las etiquetas más utilizadas son las relativas a la descripción y palabras claves, presentes en el elemento HEAD de todos los diarios con la excepción del catalán Avui. Con un nivel de presencia sensiblemente inferior se encuentran las relativas a lengua, distribución y autor.

\section{La metaetiqueta 'description'}

El contenido de esta metatag suele hacerse visible en los resultados que proporcionan ciertos buscadores. Permite conocer de forma sintética qué ofrece cada sede web de tal manera que facilita la selección del recurso más idóneo. Quince de los dieciséis diarios que han usado metatags cuentan con una destinada a la descripción sucinta del contenido de la sede web de su edición digital. La tabla III muestra la extensión en número de caracteres de cada metatag. De los datos que ofrece destaca la disparidad entre algunos medios. Si la extensión aconsejada se suele situar entre los 150 y los 200 caracteres, llama la atención tanto el volumen de las etiquetas del Diario Montañés o Ideal digital como la parquedad descrip-

\begin{tabular}{|l|c|c|}
\hline \multicolumn{1}{|c|}{ Diario } & $\mathbf{2 0 0 2}$ & $\mathbf{2 0 0 3}$ \\
\hline El Diario Montañés.es & 342 & 342 \\
\hline Ideal digital & 255 & 255 \\
\hline La Voz de Galicia & 240 & 203 \\
\hline Las Provincias digital & 209 & 132 \\
\hline El Norte de Castilla & 138 & 138 \\
\hline Faro de Vigo & 129 & 129 \\
\hline La Rioja.com & 121 & 121 \\
\hline Diario Sur digital & 120 & 120 \\
\hline Diario de Jerez digital & 97 & 97 \\
\hline DiarioVasco.com & 97 & 97 \\
\hline La Nueva España & 92 & 46 \\
\hline Canarias 7 & 62 & 62 \\
\hline Diario Información & 53 & 53 \\
\hline El Comercio digital & 39 & 94 \\
\hline El Correo digital & 18 & 18 \\
\hline
\end{tabular}

Tabla III. Número de caracteres en la metatag 'description' [Fuente: elaboración propia].

Scire. $10: 2$ (jul.-dic. 2004) 99-114. 
tiva de otros medios. El ejemplo más significativo de esto último es el laconismo de El Correo digital que resuelve el contenido de su metatag con el sintagma 'diario electrónico'. Por otro lado se reflejan también los cambios producidos: cuatro diarios han modificado sus etiquetas respecto al año 2002: tres redujeron su extensión y sólo uno la aumentó. La lengua utilizada en todos los casos es el español con la sola excepción de La Rioja digital que ha optado por el inglés. Este mismo diario es el único que actualmente no ha redactado su descripción en forma discursiva, sino que ha seleccionado un conjunto de términos más propios de la metatag 'keywords'. Con anterioridad, Las Provincias digital también había seguido un esquema semejante, pero en la revisión efectuada en 2003 lo cambió para seguir una estructura discursiva.

Al observar el contenido de esta metatag se pueden extraer ciertos datos significativos. En diez casos se hace una referencia explícita a la sede web del diario mediante expresiones del tipo: 'versión digital' (1), 'portal local' (1), 'edición digital' (2) o 'diario electrónico' (6). Esta última es la forma adoptada por la mayoría de medios del Grupo Correo. En los cinco diarios restantes sus descripciones son más genéricas y no permiten distinguir si lo que se describe es la sede web del medio o su versión impresa. Dos ejemplos pueden ilustrar este punto. Canarias 7 se presenta como "el periódico de mayor difusión y lectura de las Islas Canarias". Ideal digital se define como "un periódico de carácter regional que cubre las provincias de Granada, Jaén, Costa de Granada y Almería". La actualidad informativa es otro elemento que refleja el contenido de este tipo de metatags. Concretamente, ocho diarios han utilizado el término 'actualidad', por encima de otros con los que comparten cierta afinidad como: 'información' (4) o 'noticias' (2). Ambos términos, como se verá más adelante, tienen mayor protagonismo en la metatag 'keywords'. Por último, cabe señalar que al tratarse de diarios de ámbito local y regional, todos ellos, con la sola excepción de $E l$ Correo digital, indican de forma explícita la cobertura geográfica de su atención informativa. Existen casos de una gran precisión: en aquellos en los que el área geográfica corresponde a una comunidad autónoma se especifican las provincias (Norte de Castilla, Ideal digital, Las Provincias); en otros, como en el de La Voz de Galicia, se ha optado por incluir las localidades sobre las que existe edición.

\section{La metaetiqueta 'keywords'}

A diferencia de la metatag 'description' - que proporciona la explicación general de una sede web-, 'keywords' representa su contenido mediante la asignación de un conjunto de términos que los motores de búsqueda han utilizado para la recuperación de documentos web. Desde un punto de vista documental este proceso sería equiparable a la indización, y los términos seleccionados corresponderían a un lenguaje documental rudimentario y en nada estructurado. En este 
apartado no se pretende analizar tanto la idoneidad y oportunidad del lenguaje utilizado en cada caso, sino determinar ciertas características generales a partir de su observación. Antes de continuar conviene hacer una precisión terminológica. En adelante no se va a hablar de palabra, sino de término, entendido aquí como el conjunto de caracteres alfabéticos delimitado por el signo separador 'coma' (,). Esta precisión ha tenido una enorme importancia a la hora de determinar con exactitud tanto el número como la forma de los elementos que constituyen la base del lenguaje utilizado.

\subsection{Número y evolución de términos}

La tabla IV refleja la evolución del número de términos utilizados entre 2001 y 2003. Su crecimiento se explica por dos razones: la primera, por el aumento de diarios con 'keywords': de 13 en 2001 se pasó a 15 en 2002; y, en segundo lugar, porque su contenido también se modificó. Los datos de esta tabla sólo reflejan los términos computados en todas las etiquetas, independientemente de las veces que un mismo término haya aparecido en alguna de ellas. Podríamos hablar, entonces, de la existencia de un vocabulario base, constituido por la suma de todos los términos utilizados sin contabilizar ninguna repetición.

\begin{tabular}{|c|c|c|c|}
\hline Año & Diarios & Total términos & Vocabulario base \\
\hline 2001 & 13 & 429 & 260 \\
\hline 2002 & 15 & 523 & 330 \\
\hline 2003 & 15 & 528 & 321 \\
\hline
\end{tabular}

Tabla IV. Evolución del total de términos en la metatag 'keywords' [Fuente: elaboración propia].

La información anterior debe complementarse con la que ofrece la tabla $\mathrm{V}$ sobre la distribución de términos por metatag. Pese a los cambios a lo largo del período 2001-2003, la distribución tanto de términos totales como de términos del vocabulario base se ha mantenido estable. Si el análisis se hace sobre el contenido del vocabulario base del 2001 (260 términos), el índice de variación con respecto al 2002 fue del $44 \%$. Un año después este índice, pese a reducirse, fue del $20 \%$. Ambos datos reflejan tanto el dinamismo en la asignación del contenido de la metatag como el afán por renovarla y actualizarla. Para definir algo más el perfil del lenguaje utilizado es necesario conocer los términos con mayor frecuencia de uso, es decir, los más repetidos. Esta información queda recogida en la tabla VI, en donde aparecen los quince términos con más frecuencia de uso durante los últimos tres años. De ese conjunto, once (destacados en negrita en la tabla) han ocupado alguna de las quince primeras posiciones a lo largo de los últimos tres años. 


\begin{tabular}{|c|c|c|}
\hline Año & Total términos / metatag & Vocabulario base / metatag \\
\hline 2001 & 33 & 20 \\
\hline 2002 & $34^{\prime} 8$ & 22 \\
\hline 2003 & $35^{\prime} 2$ & $21^{\prime} 4$ \\
\hline
\end{tabular}

Tabla V. Distribución de términos por metatag [Fuente: elaboración propia].

\begin{tabular}{|c|c|c|c|c|c|}
\hline \multicolumn{2}{|l|}{2001} & \multicolumn{2}{|l|}{2002} & \multicolumn{2}{|c|}{2003} \\
\hline España & 12 & España & 10 & Periódico & 11 \\
\hline Noticias & 10 & Noticias & 10 & España & 10 \\
\hline Deportes & 8 & Periódico & 10 & Noticias & 10 \\
\hline Periódico & 8 & Información & 7 & Economía & 9 \\
\hline Publicidad & 8 & News & 7 & Deportes & 8 \\
\hline Diario & 7 & Publicidad & 7 & News & 7 \\
\hline Información & 7 & Cultura & 6 & Cultura & 6 \\
\hline Actualidad & 6 & Deportes & 6 & Fútbol & 6 \\
\hline Cultura & 6 & Economía & 6 & Información & 6 \\
\hline News & 6 & Actualidad & 5 & Opinión & 6 \\
\hline Andalucía & 5 & Andalucía & 5 & Publicidad & 6 \\
\hline El Diario Montañés & 5 & Diario & 5 & Actualidad & 5 \\
\hline Anunciante & 4 & El Diario Montañés & 5 & Andalucía & 5 \\
\hline Economía & 4 & Fútbol & 5 & Juegos & 5 \\
\hline Euskadi & 4 & Prensa & 5 & Música & 5 \\
\hline
\end{tabular}

Tabla VI. Términos con mayor frecuencia de uso [Fuente: elaboración propia].

Más de la mitad del total de términos aparece en la página inicial de la sede web del diario. Parece que en buena medida la página inicial constituye una fuente de información esencial para seleccionar los términos más habituales, o bien, que el contenido de la metatag refleja la estructura de la página inicial del diario. La figura 2 muestra la consolidación de esa tendencia con el ligero crecimiento que se ha ido produciendo en los últimos tres años. Este dato cobra mayor relieve cuando se tiene en consideración que a lo largo del período de referencia (2001-2003) el 42\% del total de términos aparecen en la página de inicio y que este porcentaje se eleva al $54 \%$ cuando los términos analizados son los de mayor frecuencia.

La cuestión que cabe dilucidar ahora es conocer el perfil temático de los términos que conforman el contenido de la metatag. Aquí se va a distinguir, como se ha hecho anteriormente, entre los términos que aparecen o no en la página de inicio de la sede web del diario.

Scire. 10 : 2 (jul.-dic. 2004) 99-114. 


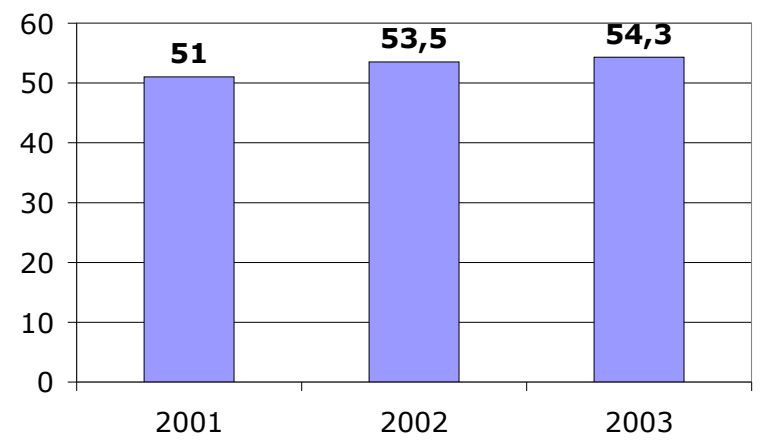

Figura 2. Porcentaje de términos con mayor frecuencia de uso presentes en la página de inicio [Fuente: elaboración propia].

\subsection{Términos presentes en la página de inicio}

En la tabla VII se muestra la distribución de todos los términos computados durante el período 2001-2003 agrupados en seis categorías. Más de la mitad hacen referencia a las secciones periodísticas habituales y suelen formar parte del sumario en el que se estructuran los contenidos informativos del medio. La siguiente categoría corresponde a las designaciones del propio medio, o a otros del mismo grupo editorial a los que se remite desde la página de inicio. La tercera, aunque con un porcentaje significativamente menor, refleja la variada oferta de servicios, tanto de acceso a información retrospectiva, como lúdica o de comunicación. De forma un tanto marginal cabe señalar la presencia de topónimos referidos habitualmente al área geográfica que el medio suele cubrir informativamente.

\begin{tabular}{|l|c|c|}
\hline \multicolumn{1}{|c}{ Categoría } & Núm. términos & Porcentaje \\
\hline Secciones periodísticas & 341 & $54,6 \%$ \\
\hline Medios y grupos editoriales & 153 & $24,5 \%$ \\
\hline Servicios & 80 & $12,8 \%$ \\
\hline Topónimos & 31 & $4,9 \%$ \\
\hline Temas informativos & 10 & $1,6 \%$ \\
\hline Entidades & 9 & $1,4 \%$ \\
\hline
\end{tabular}

Tabla VII. Distribución de términos presentes en la página de inicio por categorías [Fuente: elaboración propia].

Los datos recogidos en la tabla VII se deben interpretar a la luz de la figura 3 que da cuenta de las variaciones producidas en las diferentes categorías en que se han agrupado los términos a lo largo de los tres últimos años. De esta manera se observa cómo progresivamente ha aumentado el peso tanto de las secciones 


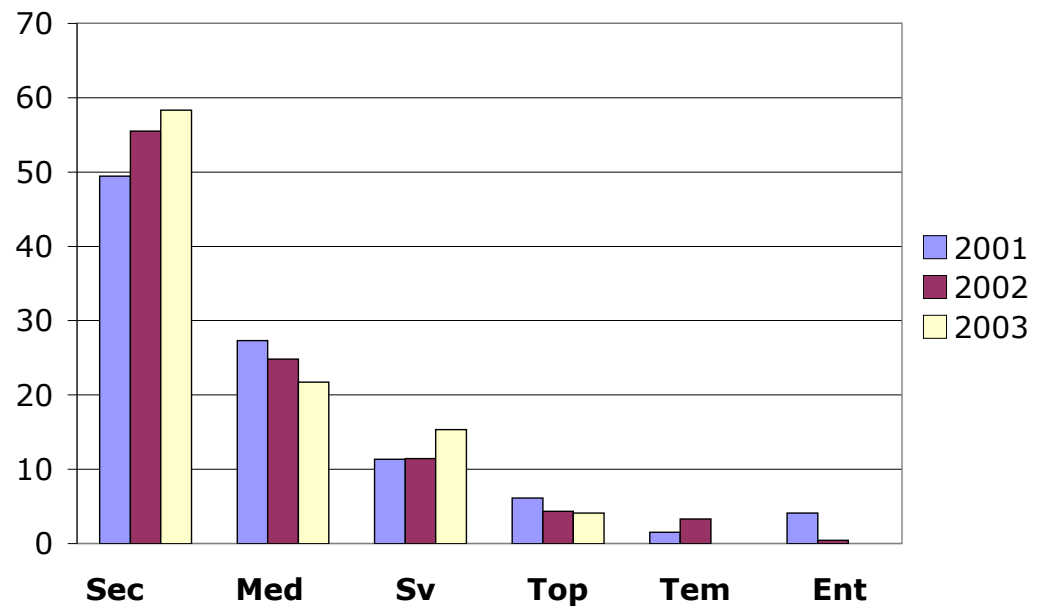

Figura 3. Evolución de los términos presentes en la página de inicio durante el período 2001-2003 [Fuente: elaboración propia].

periodísticas (9\%) y de los servicios (4\%), mientras que el resto de las categorías ha disminuido. De ello se desprende el interés de reflejar en la metatag tanto los contenidos informativos de forma general a través de la enumeración de las secciones periodísticas como la creciente oferta de servicios que enriquece y hace atractiva la sede web de un medio de comunicación.

\subsection{Términos no presentes en la página de inicio}

La tabla VIII recoge la distribución de términos que no aparecen en la página de inicio de las sedes web de los diarios. Están agrupados en siete categorías. Las más numerosas corresponden, por este orden, a los términos toponímicos y a los referidos a temas informativos. La importancia de los topónimos viene determinada por el ámbito local y regional de cada medio. En cuanto a los temas, pese a su heterogeneidad, destacan dos subcategorías. Por un lado, términos que reflejan aspectos de la realidad sociocultural del entorno geográfico que cubre el medio, y por otro, en un grado menor, disciplinas deportivas. La tercera categoría incluye designaciones genéricas relativas a la información de actualidad y a los medios de comunicación. Entre las entidades, las deportivas - especialmente clubes de fútbol - tienen un peso destacado. Un lugar menor lo ocupan entidades públicas de carácter gubernamental. Por último, cabe señalar el papel significativamente menor que ocupan términos relacionados tanto con las secciones periodísticas como con la oferta de servicios de la prensa digital. En la mayoría de los casos se trata de términos sinónimos a los presentes en la página de inicio del diario o a su equivalente en inglés. 


\begin{tabular}{|l|c|c|}
\hline \multicolumn{1}{|c|}{ Categoría } & Núm. términos & Porcentaje \\
\hline Topónimos & 243 & $28,3 \%$ \\
\hline Temas informativos & 232 & $27,0 \%$ \\
\hline $\begin{array}{l}\text { Designaciones genéricas relativas a informa- } \\
\text { ción de actualidad y medios de comunicación }\end{array}$ & 158 & $18,4 \%$ \\
\hline Secciones periodísticas & 90 & $10,5 \%$ \\
\hline Entidades & 72 & $8,4 \%$ \\
\hline Servicios & 51 & $5,9 \%$ \\
\hline Personas & 11 & $1,2 \%$ \\
\hline
\end{tabular}

Tabla VIII. Distribución de términos no presentes en la página de inicio por ámbitos temáticos [Fuente: elaboración propia].

Tal y como se ha hecho al hablar de los términos presentes en las páginas de inicio, conviene complementar el valor informativo de la tabla VIII con los datos que refleja la figura 4. En ella destacan dos aspectos: en primer lugar, el uso creciente de topónimos, claro reflejo del ámbito informativo de los medios locales; $y$, en segundo lugar, el aumento constante de términos relativos a la oferta de servicios, lo que parece revelar la importancia otorgada por los medios digitales a la capacidad de interactuar con su audiencia.

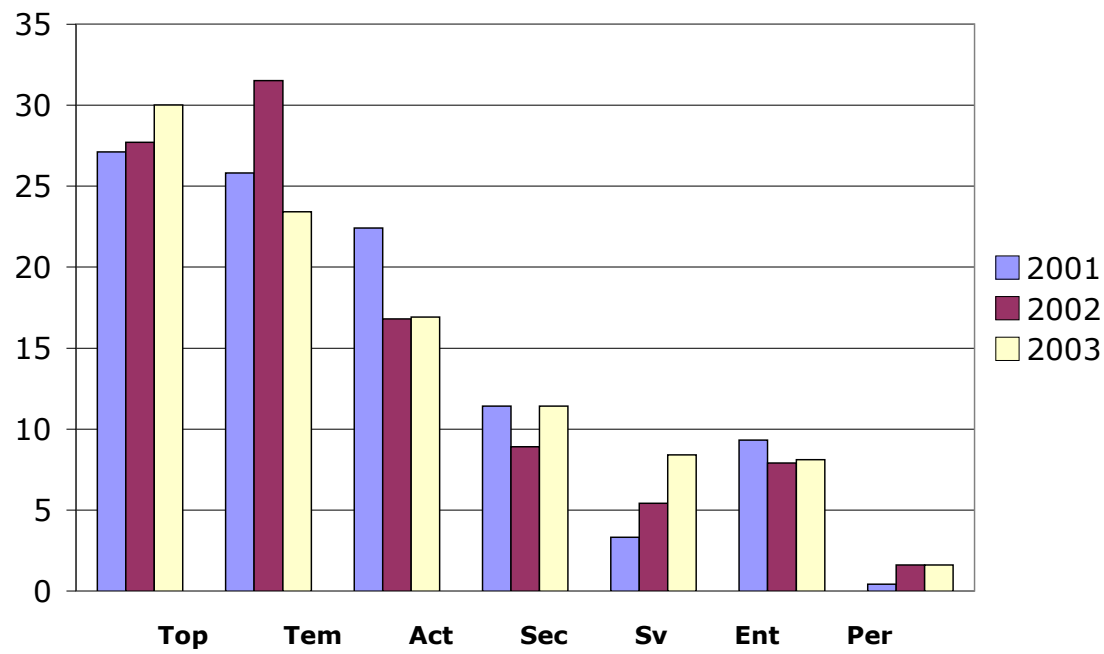

Figura 4. Evolución de los términos no presentes en la página de inicio durante el período 2001-2003 [Fuente: elaboración propia].

Scire. $10: 2$ (jul.-dic. 2004) 99-114. 


\subsection{Términos coyunturales}

La función de la metatag 'keywords' tiene como objetivo representar la sede web del diario y no tanto dar cuenta de la actualidad periodística. Cuando ciertos términos traducen la actualidad informativa hablamos de términos coyunturales. Consideramos que su uso responde a una estrategia orientada a mejorar la visibilidad del medio. Estrategia desaconsejable puesto que los términos están sujetos al nivel de obsolescencia propio del hecho noticioso. De este modo la ausencia de una actualización periódica de la metatag limita la utilidad de este tipo de términos. Algunos ejemplos ilustrarán mejor este punto. Norte de Castilla todavía incluye términos como 'antenas de telefonía' o 'Gescartera', que remiten a temas que en su momento ocuparon la atención de la opinión pública, pero que han sido relegados, porque su vigencia informativa es prácticamente nula. Lo mismo sucede con aquellos medios que han incluido nombres de personajes públicos de la vida política o deportiva.

\subsection{Diversidad lingüística}

Para concluir el repaso al perfil del contenido de la metatag 'keywords' utilizada por los diarios locales y regionales es necesario hacer referencia a la selección de términos en otras lenguas al margen del español. La asignación de términos en otras lenguas que no sea el español persigue también aumentar la visibilidad de los medios. El inglés es la lengua utilizada en la práctica totalidad de los casos. La tabla IX —en la página siguiente - muestra la distribución de este tipo de términos entre los diversos diarios a lo largo del período 2001-2003. Se observa un significativo crecimiento con el paso del tiempo. Este hecho viene a demostrar la conciencia cada vez más clara de adecuar el contenido de la etiqueta a la lengua franca de la Red y contribuir a la visibilidad del medio. Es reseñable la estrategia de diarios como La Rioja.com (73\%) y Las Provincias (53\%) de asignar la mayoría de los términos en inglés. Por otro lado, destaca que una tercera parte de los diarios (5) no haya incluido ningún término en otra lengua que no sea el español. Todo ello muestra la disparidad de criterios entre los diarios.

En la figura 5 se puede observar la evolución a la que se ha hecho referencia haciendo hincapié ahora en las categorías temáticas en las que se agrupan los términos en lenguas extranjeras. Por un lado se evidencia el crecimiento constante en todas las categorías. Destacan las tres principales que son las que configuran la base de la representación sintética de los medios locales en la Red fuera de nuestro entorno lingüístico y cultural, es decir, a través de la designación genérica relativa a la información de actualidad, la cobertura informativa atendiendo a criterios geográficos y por último a la oferta de servicios propios del entorno digital. 
112 Àngels Jiménez, Eulàlia Fuentes y Alfons González

\begin{tabular}{|l|c|c|c|}
\hline \multicolumn{1}{c}{ Diario } & 2001 & 2002 & 2003 \\
\hline Las Provincias digital & 1 & 17 & 17 \\
\hline La Rioja.com & 11 & 11 & 11 \\
\hline La Voz de Galicia & 9 & 9 & 7 \\
\hline Canarias 7 & 7 & 7 & 7 \\
\hline DiarioVasco.com & - & 7 & 7 \\
\hline El Comercio digital & - & - & 7 \\
\hline El Diario Montañés.es & 1 & 1 & 4 \\
\hline El Norte de Castilla & 1 & 1 & 1 \\
\hline Diario de Jerez digital & 1 & 1 & 1 \\
\hline Faro de Vigo & - & 1 & 1 \\
\hline El Correo digital & - & - & - \\
\hline Diario Sur digital & - & - & - \\
\hline Ideal digital & - & - & - \\
\hline La Nueva España & - & - & - \\
\hline Diario Información & - & - & - \\
\hline \multicolumn{1}{|c|}{ Total } & 31 & 39 & 63 \\
\hline
\end{tabular}

Tabla 9. Distribución de términos extranjeros durante el período 2001-2003 [Fuente: elaboración propia].
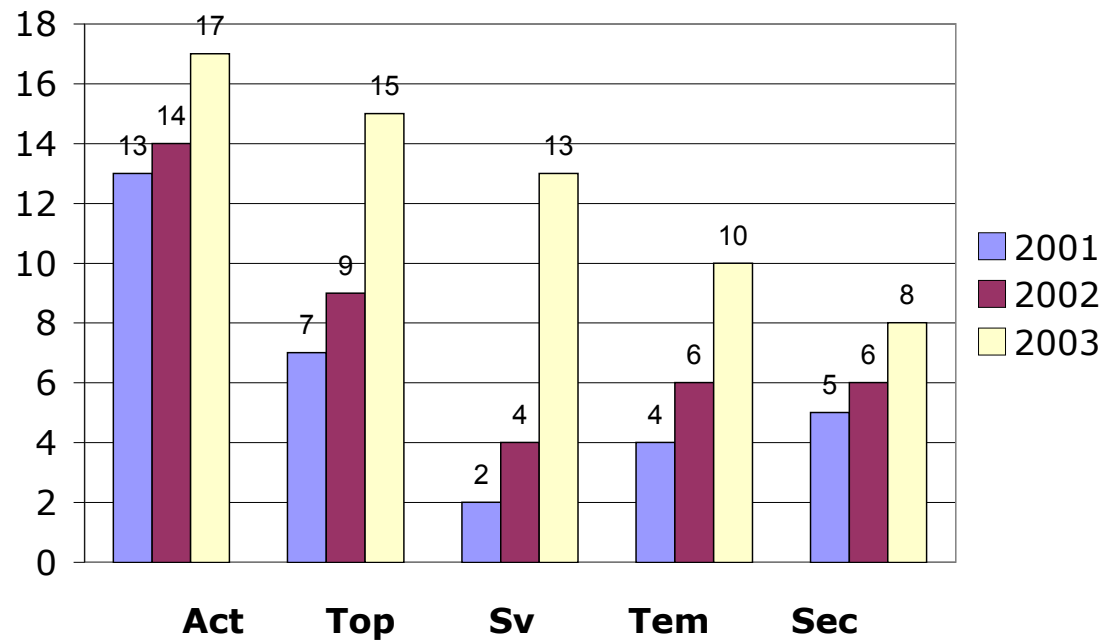

Figura 5. Evolución de los términos extranjeros durante el período 2001-2003 [Fuente: elaboración propia].

Scire. $10: 2$ (jul.-dic. 2004) 99-114. 


\section{Conclusiones}

A tenor de lo expuesto en los apartados precedentes y de forma sintética se pueden esbozar varias conclusiones. El lenguaje HTML no ha contribuido a la existencia de un modelo normalizado de descripción, y prueba de ello es la heterogeneidad tanto en el número como en el tipo y en el contenido de las metatags presentes en la sede web de cada diario analizado. A pesar del 'desinterés' de los motores de búsqueda más populares en la Red hacia el contenido de las metatags, un buen número de los diarios analizados las ha actualizado en los últimos años al menos en una ocasión.

En lo que concierne a la metatag 'keywords', parece que la página inicial de la sede web del medio constituye una fuente de información esencial para seleccionar los términos con mayor frecuencia de uso. Asimismo refleja la estructura de los contenidos informativos del diario y la oferta de servicios de valor añadido, es decir, capacidad interactiva.

Entre las estrategias para aumentar la visibilidad no sólo cabe citar la actualización periódica del contenido de la metatag 'keywords', sino también el uso de términos coyunturales y la creciente inclusión de términos en inglés para adecuarse a la lingua franca de la Red.

\section{Referencias}

Codina, Lluís (1997). Cómo funcionan los servicios de búsqueda en Internet: un informe especial para navegantes y creadores de información (parte I). // Information World en Español. 6:5 (mayo 1997) 22-27.

A Dictionary of HTML metatags [documento HTML]. Vancouver Webpages, rev. 16 septiembre 2003. URL: <http://andrew.triumf.ca/VW/META/>.

Jiménez, Àngels. (2001). Estudi de la gestió documental de la informació en els serveis de valor afegit dels mitjans de comunicació a Internet: el cas de la premsa diària a l'Estat espanyol [en línea]. Barcelona: CBUC: CESCA, 2001. Tesis doctoral. URL: <http: //www.tdcat.cesca.es/TDX-1008102-143437>.

Méndez Rodríguez, Eva. (2002). Metadatos y recuperación de información: estándares, problemas y aplicabilidad en bibliotecas digitales. Gijón: Trea, 2002. (Biblioteconomía y administración cultural). 